\title{
Controller design for Robotic hand through Electroencephalogram
}

\author{
P. Pandelidis ${ }^{1, a}$, N. Kiriazis ${ }^{2}$, K. Orgianelis ${ }^{3}$ and N. Koulios ${ }^{4}$ \\ 1,3,4 students at the Department of Automation Eng. Sterea Ellada Institute of Technology, 34400 Psahna Evias, Halkis, Greece \\ ${ }^{2}$ MSc students at the Department of Automation Eng. Sterea Ellada Institute of Technology, 34400 Psahna Evias, Halkis, Greece
}

\begin{abstract}
This paper deals with the designing, the construction and the control of a robotic hand via an electroencephalogram sensor. First a robotic device that is able to mimic a real human hand is constructed. A PID controller is designed in order to improve the performance of the robotic arm for grabbing objects. Furthermore, a novel design approach is presented for controlling the motion of the robotic arm using signals produced from an innovative electroencephalogram sensor that detects the concentration of the brain
\end{abstract}

\section{INTRODUCTION}

The existing methods of controlling a robotic hand are the sensor placement into the neurons of the arm. Surgery for the person with a crippled hand is necessary. So we tried to find a way to obviate the need of surgery. We used the EEG sensor. The desired outcome would be the ability of closing the fingers and rotating the wrist of the hand with concentration. More specifically when the "user" wants to grab an object, the physical reaction of his mind is to concentrate to that object. Then the EEG sensor communicates with the hand "telling" it to close and grab the object.

The robotic hand mimics the movements of a real human hand since it consists of five fingers and a wrist. Each one of the fingers i.e. the thumb, the index, the ring finger, the middle finger and the small has three degrees of freedom. The wrist adds one more degree of freedom in hand which can permits the movement of the hand in the y axis.[1], [6], [7] and [8]. EEG sensor is the basic part of the control system. The sensor is a device called mindflex and interfaced with an avr microcontroller. The inputs of the system during operation are the electroencephalogram (EEG sensor) in order to achieve full control of the bionic hand. The EEG sensor performs an electroencephalogram and enables the user to view the concentration values of his mind on a monitor in real time. This data is used for controlling the servo motors of the bionic hand. The operation of the bionic arm is based on the existence of signals produced from mind, and the position of the fingers of the robot hand track the desired mind concentration values [1]-[8].

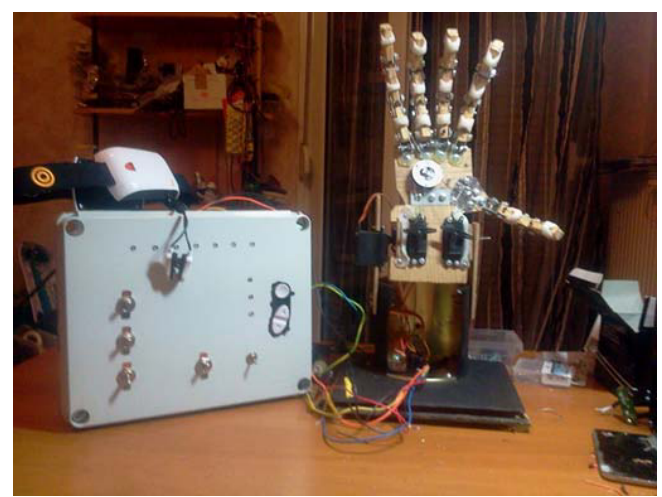

Also a ribbon soft potentiometer (pressure sensor) was placed on the palm of the robotic hand. The measured values of this sensor are the feedback of the PID controller. We used this sensor with the PID controller to prevent situations in which the object, which is caught by the robotic hand, tends to fall. The sensor detects the pressure exerted by the object located in the handle of the hand. In more details the PID controller receives feedback from the values of the pressure sensor (variations in pressure that the object exerts on the robotic hand). If a set - point is specified the PID controller regulates the servomotors by sending PWM signals to rotate them properly and catches the object effectively. In order to make the operation of the controller more understandable but also to see how it behaves in real time, we have built a GUI for the process. In this graphic environment we can observe the desired value, the sensor value and the output of the PID controller. We can also adjust the parameters of the PID controller in real time and observe how the output of the PID controller behaves [1]-[11]

\footnotetext{
${ }^{a}$ Corresponding author: rockytelis@hotmail.com
} 


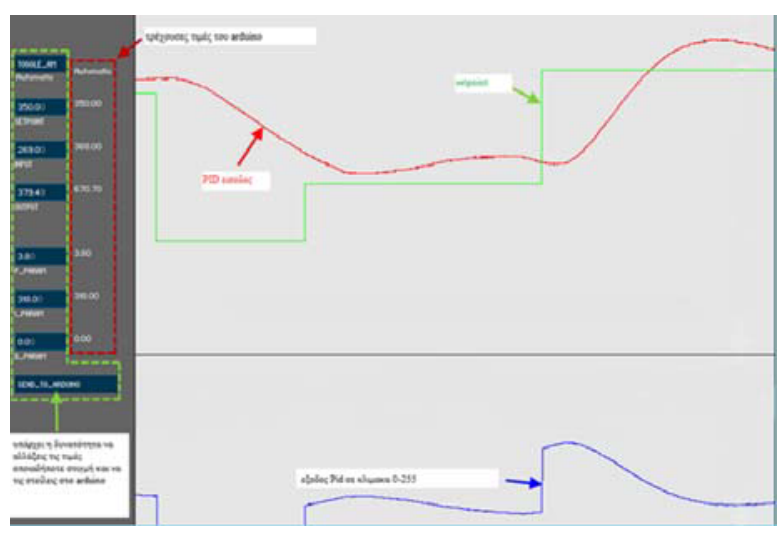

\section{ROBOTIC HAND CONSTRUCTION}

The use of robotic systems as prosthetics can alleviate the limitations arising from the amputation of the upper limbs, thus reducing the disproportionately high social and economic consequences for the victims (constraints to personal hygiene, social interaction and restrictions on occupational activities). The majority of these cases are treated by passive prosthetics contributing to a cosmetic effect offering a rudimentary level of functionality. But even the most modern commercial prosthetic hands are unable to fully meet the needs of their users, and their cost is at a very high level. The construction of the robotic hand requires experimentation in the selection of appropriate materials, the design part, the dimensions and weight of each link of the robot. The basic desired characteristics are the stability of the handle and the response speed of the joints. The robotic hand consists of 16 joints and 6 degrees of freedom (DOF). Each finger has three joints and there is one more joint that of the wrist. Three actuators are used. In order to reduce the number of actuators while achieving movement of all fingers a hybrid mechanism is used. [1], [6], [7] and [8].

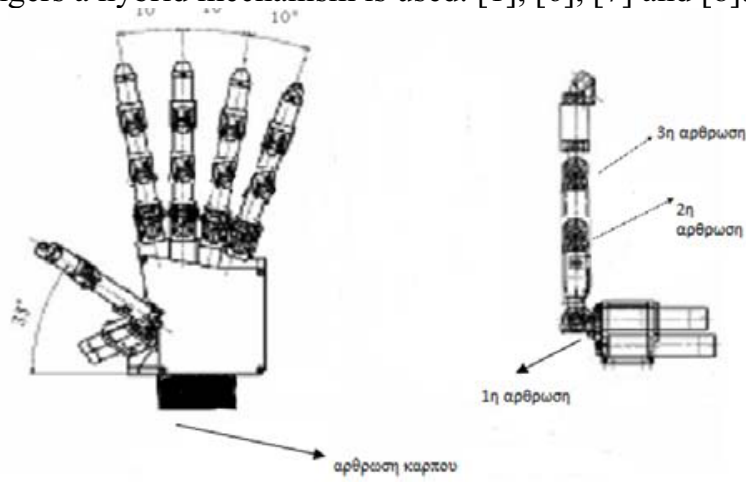

The mechanism includes a pulley and a bar to achieve movement of the thumb. At the end of each finger there is a wire - line which crosses over the entire finger and reaches the servo motor. The movement of the finger is achieved by the rotation of the servo motor which pulls the finger. The robotic arm is made of $90 \%$ wood.

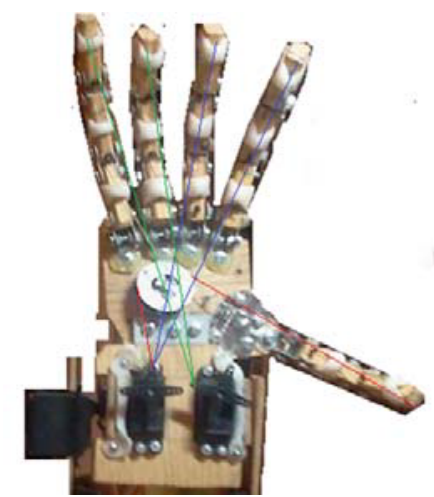

Metal clamps and galvanized screws of $4 \mathrm{~mm}$ are used to construct the joints of the robotic hand. A raw material of plastic (polymorph)also used. When this polymorph is exposed to temperatures of $60^{\circ} \mathrm{C}$ it becomes pliable and therefore we can construct plastic structures according to the requirements of the construction. The robotic hand uses three servomotors of $5.2 \mathrm{~kg} * \mathrm{~cm}$ torque to close all the fingers. The range of rotation is $180^{\circ}$. The servo motors have potentiometers which help us to detect the angular displacement. A servo motor consists of a DC motor, a potentiometer, tuning components and an intelligent circuit. The intelligent circuit with the potentiometer enables the servo motor to rotate according to our requirements. As it is known, a small DC motor has a high rotation speed at low torque, a torque not enough to move even a light load. In the servo motor, the gear unit will transform a high speed input to a lower output speed, thus more practical and widely applicable [7] and [8].

The robotic hand is fully controlled by a central control unit. There is a microcontroller which receives input from the EEG sensor and the ribbon soft potentiometer (pressure sensor). It has three outputs (the three servo motors). The EEG sensor has its own microcontroller and a wireless rf transmission system in order to send the data from the wearable sensor to the central unit of the microcontroller. We designed and built circuits that connect data from the microcontroller of the sensor to the avr microcontroller which we have programmed. Furthermore we built circuits for providing external power supply for the servo motors. The control system is in the central control unit on which there are buttons that control the power supply to the servo motors and the power supply to the microcontroller of the sensor [4] and [5].

\section{ROBOTIC HAND CONTROL}

EEG sensor record changes in the electric potential of the extracellular matrix of the cortex of the brain, i.e. changes in the number of cargo space which has bark: when active nerve cells of the cortex then move consignments to or from the extracellular space, thus changing the number of consignments of the extracellular matrix, thus changing the potential.

The signal of the EEG may be straight-horizontal- or have lines that deviate from this level. The straight horizontal line is called isoelectric line. It is named isoelectric because its duration does not change the 
potential of the extracellular matrix that is recorded in the electrode, which means that there will be no current passing from the membrane, hence the nerve cells are at rest. The lines that diverge from the isoelectric line are called ridges and show that changed the potential of the extracellular space of the brain that is initiated that stream through the membrane of the nerve cell that is activated to nerve cells (not in the idle state). Thus the waves are indicative of electrical activity of nerve cells in the cortex. The waves may be positive, i.e. above the isoelectric line or negative i.e. below the isoelectric line, depending on whether the positive charges move to or from the recording electrode [4] and [5].
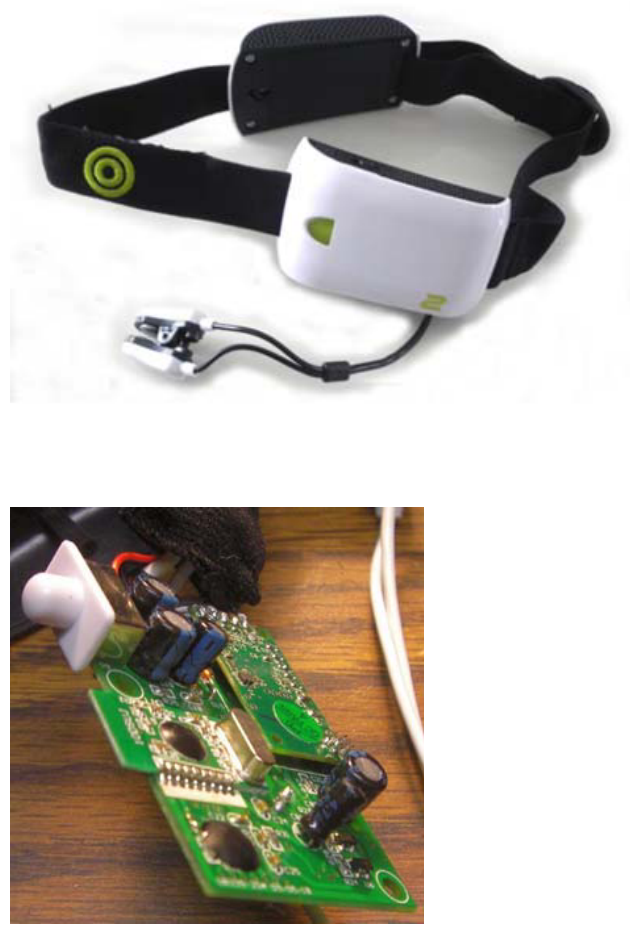

Basic waveforms are the alpha, beta, theta and delta rhythms. Alpha waves occur at a frequency of 8-12 cycles per second at a normal rate. They are present only when you are awake but have your eyes closed. They usually disappear when you open your eyes or start mental concentration. Beta waves occur at a frequency of 13 to 30 cycles per second. They are commonly associated with anxiety, depression, or the use of sedatives. Theta waves occur at a frequency of 4-7 cycles per second. It is most common in children and young adults. Delta waves occur at a frequency of 0.5 to 3.5 cycles per second. They usually occur only in young children during sleep [4] and [5].

\section{EEG brainwaves}

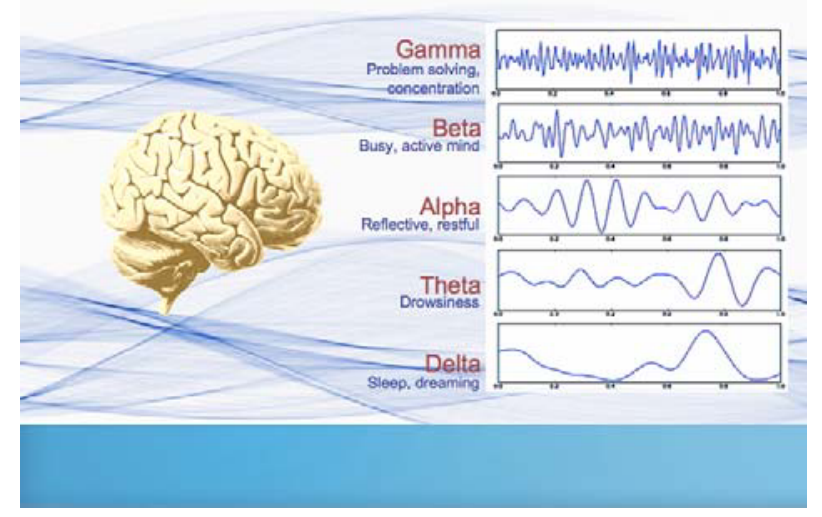

Our sensor detects small voltage fluctuations, amplifies them and feeds to the signal processing algorithm of Neurosky's microcontroller. The signal processor removes any signal which is external noise. Then sends the signal to a series of transit bandwidth (band-pass filters) where the signal is split into different categories of brain waves depending on the frequency delta, theta, high and low alpha, beta, and gamma waves. The serial output of the controller has a channel for each of the types of brain waves as attention and concentration. The channels of attention and concentration are based on an analysis of the different types of waves. The channel of concentration is fed to our microcontroller and is calibrated. This signal controls the servomotors.

The operation of the robotic hand is as follows: 1) The user of the robotic hand approaches an object to the hand at a suitable distance in order for the hand to be able to catch it. 2) The user then concentrates. 3) Once he is concentrated enough the servomotors rotate properly so as to close the hand and grasp the object. At this point the problem of a bad grip of an object had to be addressed.

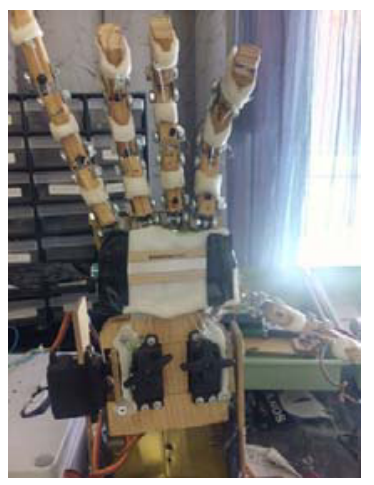

For this reason we used a PID controller and a pressure sensor (ribbon soft potentiometer). The sensor is placed on the palm of the robotic hand and detects the pressure exerted by the object located in the handle of the hand [3]. 

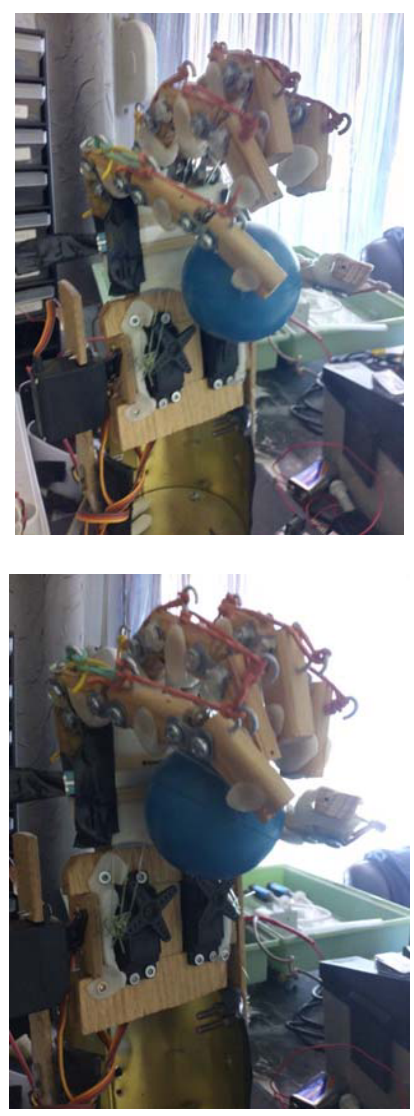

The PID controller uses the signal of the sensor for feedback purposes. The signal is an analog one of $0-5 \mathrm{~V}$ that is fed to the microcontroller. It is then converted from analog to digital, as it constitutes one of the two inputs of the system. The Error variable is the difference between the desired value (Set point) and the measured value derived from the output of the system. The Error variable is the input of the PID controller. The output of the controller drives the robotic hand at each time instant [3].

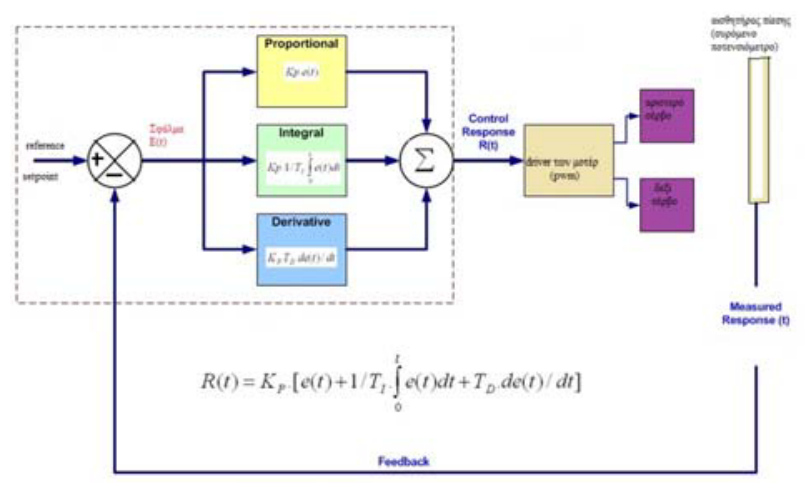

\section{CONCLUSIONS}

In this paper a design of a robotic bionic hand is presented. Also an electroencephalogram is applied for the control of the robot through the concentration of the mind. A PID controller is proposed. PID controls the position of servo motors and prevents situations in which the object which is caught by the robotic hand tends to fall.
The robotic hand has been tested in real conditions in order to tune the PID controller so that the system becomes operational. When the user concentrates, the fingers of the robotic hand close and an object is caught. The sensor which is fitted on the palm of the robot hand sends data to the PID controller. Also we supply the PID controller with a desired output value. During operation, servo motors rotate to a desired value (measured in degrees). We thus achieve a reliable grip of an object from the robotic hand.

\section{REFERENCES}

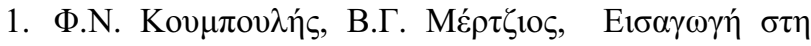

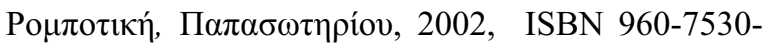
13-6

2. P. N. Paraskevopoulos, Digital Control Systems, New York : Prentice Hall, 1996

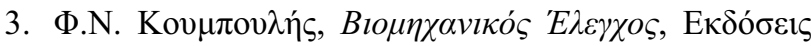

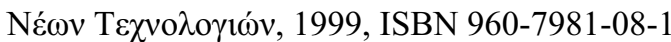

4. http://openeeg.sourceforge.net/doc/

5. Handbook of EEG Interpretation, Second Edition (William Tatum IV DO)

6. M. Tsiantakis, Graduation Robonova, TEI Crete Department of Mechanical Engineering, 2012.

7. Craig John J. Introduction to Robotics Introduction and Self-Control [Book]: Prentice Hall, 2009.

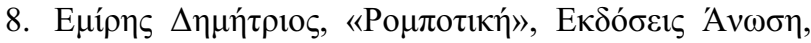
1999

9. M. G. Skarpetis, and F. N. Koumboulis, "Robust PID Controller for Electro - Hydraulic Actuators, 18th IEEE International Conference on Emerging Technologies and Factory Automation (ETFA2013) September 10-13, 2013, Cagliari, Italy

10. M. G. Skarpetis, F. N. Koumboulis, and A. S. Ntellis, Robust Control Algorithms for a Hydraulic Actuator with Variable Displacement Vane Pump, 17th Conference on Emerging Technologies \& Factory Automation (ETFA), September 17-21, 2012, Kraków, Poland

11. A M. G. Skarpetis, F. N. Koumboulis and A.S. Ntellis Heuristic Control Algorithm for Robust Internal Model Control with Arbitrary Reference Model, , J.L. Ferrier et al. (eds.), Informatics in Control, Automation and Robotics, Lecture Notes in Electrical Engineering 283, 2013 